\title{
Observation of Anomalous Spin Segregation in a Trapped Fermi Gas
}

\author{
X. Du, L. Luo, B. Clancy, and J. E. Thoma:* \\ Duke University, Department of Physics, Durham, North Carolina, 27708, USA
}

(Dated: October 25, 2018)

\begin{abstract}
We report the observation of spin segregation, i.e., separation of spin density profiles, in a trapped ultracold Fermi gas of ${ }^{6} \mathrm{Li}$ with a magnetically tunable scattering length close to zero. The roles of the spin components are inverted when the sign of the scattering length is reversed. The observed density profiles are in qualitative agreement with the spin-wave theory applied previously to explain spin segregation in a Bose gas, but disagree in amplitude by two orders of magnitude. The observed atomic density profiles are far from equilibrium, yet they persist for $\simeq 5$ seconds in a trap with an axial frequency of $\simeq 150 \mathrm{~Hz}$. We attribute this long lifetime to Fermi statistics: The scattering amplitude is nonzero only for atoms in opposite states, and vanishes for atoms in the same state. By measuring the magnetic field at which spin segregation ceases, we precisely determine the zero crossing in the scattering length of ${ }^{6} \mathrm{Li}$ as $527.5 \pm 0.2 \mathrm{G}$.
\end{abstract}

Ultracold Fermi gases near a Feshbach resonance [1] exhibit strong interactions [2] and offer unprecedented opportunities to test nonperturbative quantum many-body theory in systems from high temperature superconductors to nuclear matter. Near the resonance, a bias magnetic field tunes the interaction between two spin states from strongly repulsive to strongly attractive. These strongly interacting Fermi gases have been extensively studied over the last few years [3].

In contrast, the weakly interacting regime of a Fermi gas near a Feshbach resonance has received relatively little attention. For a bias magnetic field near the zero crossing, the s-wave scattering length for atoms in opposite spin states can be tuned smoothly from small and positive to small and negative as the bias field is varied.

We report the observation of spin segregation in an optically-trapped ultracold Fermi gas of ${ }^{6} \mathrm{Li}$ with a bias magnetic field close to the zero crossing. A sample of ultracold ${ }^{6} \mathrm{Li}$ gas is created in a coherent superposition of the two lowest spin states $|1\rangle$ and $|2\rangle$, with equal population in each state. Initially, the two spin states have the same atomic density profile. After several hundred ms, the spatial densities segregate, with one spin state moving outward and one moving inward, as shown in Fig. 1. The roles of the spin states are interchanged when the sign of the scattering length is reversed. The differential trapping potential experienced by atoms in the two states cannot account for the spin segregation, since it is four orders of magnitude smaller than the thermal energy $k_{B} T$. A longitudinal spin wave theory $[4,[5,[6]$ has been used to explain spin segregation that was observed in a trapped Bose gas [7]. In the Bose gas, numerical simulations are in good quantitative agreement with the experimental results. In contrast, our observations of spin segregation in a Fermi gas show great discrepancy with the theoretical calculations based on a spin wave assumption.

In the experiments, a sample of ${ }^{6} \mathrm{Li}$ atoms in a $50 / 50$ mixture of the two lowest hyperfine states is loaded into a $\mathrm{CO}_{2}$ laser trap with a bias magnetic field of $840 \mathrm{G}$, where the two states are strongly interacting. Evapora-

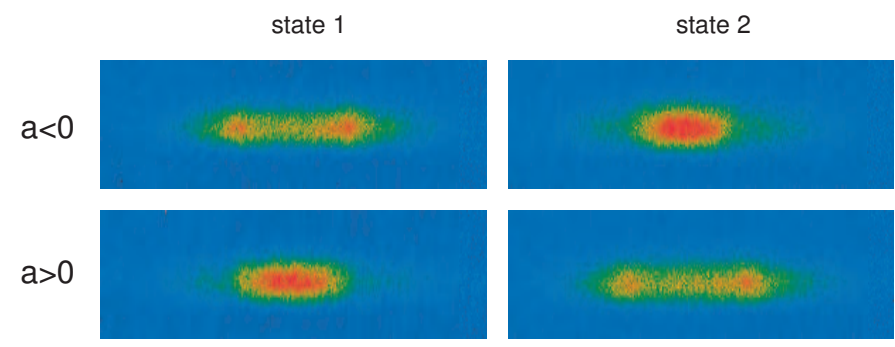

FIG. 1: Absorption images (state 1 and state 2) taken at $200 \mathrm{~ms}$ after the RF pulse for $526.2 \mathrm{G}$ (scattering length $a<$ $0)$ and for $528.8 \mathrm{G}(a>0)$. Each image is $1.2 \mathrm{~mm}$ in the horizontal direction.

tive cooling is performed to lower the temperature of the sample [2]. The magnetic field is then increased in 0.8 seconds to a weakly interacting regime at $1200 \mathrm{G}$ where an on-resonance optical pulse of $15 \mu \mathrm{s}$ is applied to remove atoms of one state while leaving atoms in the other state intact. With the single state present, the magnetic field is lowered in 0.8 seconds to $528 \mathrm{G}$, near the zero crossing. Then an RF pulse is applied on the $|1\rangle-|2\rangle$ transition to create spin coherence. Instead of applying a $\pi / 2$ pulse, the RF frequency is swept across the resonance of the hyperfine transition $\sim 75.60 \mathrm{MHz}$ to achieve the coherent spin transfer. We control the amplitude of the spin transfer by varying the sweep rate. Typically, we sweep the RF frequency by $35 \mathrm{kHz}$ (centered at hyperfine transition) in $40 \mathrm{~ms}$ in order to transfer about $50 \%$ of the atoms. Finally, we take absorption images of atoms in both states (in separate experimental cycles) at various times after the RF pulse. From those images, we can determine the parameters of the cloud sample such as temperature and density. At the final optical trap depth, the measured trap oscillation frequency in the transverse directions is $\omega_{\perp}=2 \pi \times 4360 \mathrm{~Hz}$, while the axial frequency is $\omega_{z}=2 \pi \times 145 \mathrm{~Hz}$ at $528 \mathrm{G}$. Atoms in each of the two spin states experience slightly different magnetic trap potentials in the bias magnetic field. Due to the bias magnetic field curvature, the differential potential $U_{1}-U_{2}$ varies 
across the sample and $d^{2}\left(U_{1}-U_{2}\right) / h / d z^{2}=4.4 \times 10^{-4}$ $\mathrm{Hz} / \mu \mathrm{m}^{2}$. The total number of atoms is $N \simeq 2.0 \times 10^{5}$. The corresponding Fermi temperature is $T_{F} \simeq 7 \mu \mathrm{K}$.

We find that the spin segregation is not sensitive to the temperature of the cloud. We observed spin segregation over the range from a degenerate Fermi gas $\left(T \simeq 0.15 T_{F}\right)$ to a non-degenerate one $\left(T \simeq 4 T_{F}\right)$. All the data shown in this Letter were taken in the non-degenerate regime.

The experimental results for spin segregation as a function of bias magnetic field are summarized in Fig. 2, Here, the sample temperature is $T \simeq 27 \mu \mathrm{K}$ and the peak atomic density is $1.2 \times 10^{12} / \mathrm{cm}^{3}$. The axial $1 / \mathrm{e}$ width for a fit of a gaussian distribution to the initial density profile of the sample is $\simeq 300 \mu \mathrm{m}$.

We use the data at $529.3 \mathrm{G}$, Fig. 2, as an example to describe the observed spin segregation in our experiments. Note that magnetic fields are calibrated with an RF spectroscopic technique, as described below. Spin segregation occurs only after a coherent superposition of the two states is created. Spin segregation starts to build up during the RF pulse duration of $40 \mathrm{~ms}$, as we sweep the RF frequency across the hyperfine transition. Starting about $40 \mathrm{~ms}$ after the RF pulse, we observe a significant change in the spatial profiles of the initially overlapping clouds for states $|1\rangle$ and $|2\rangle$. The cloud size of state $|1\rangle$ decreases while the density profile of state $|2\rangle$ evolves into a double-peak structure. Maximum segregation occurs at about $200 \mathrm{~ms}$ and is maintained for several seconds. Then the two segregated clouds relax back to their original overlapping profiles in $\sim 9$ seconds.

Spin segregation observed in an ultracold Bose gas 7 ] has been described previously as overdamped spin wave [4, 5, 6]. The basic idea can be described in terms of a Bloch vector. We take the axis $w$ as the "longitudinal" population inversion, and the axes $u$ and $v$ as the "transverse" coherence. An RF pulse prepares a coherent superposition of spin-up and spin-down with polarization in the $u-v$ plane. Due to the magnetic field inhomogeneity, the hyperfine transition frequency varies along the long axial direction of the sample $\sim 10 \mathrm{~Hz}$. After the RF pulse, the inhomogeneous precession of the Bloch vectors about the $w$-axis starts to build up a spin orientation gradient in $u-v$ plane along the axial direction. Meanwhile, atoms with different spins move around and collide with each other. The interaction due to the binary collision leads to precession of each atom's spin about the total spin vector of both atoms. Since both atoms have spins in $u-v$ plane, the precession of each spin about the total spin in $u-v$ plane produces spin components out of the $u-v$ plane. The subsequent formation of a $w$ component leads to a spatially varying population inversion and accounts for the spin segregation.

We tried to use a spin-wave theory to explain the spin segregation observed in our experiment. We derived equations for the spin density and spin current using a Wigner function operator approach [10]. The Wigner phase space operators are written in terms of position space creation and annihilation operators

$\hat{W}_{\alpha \alpha^{\prime}}(\mathbf{r}, \mathbf{p}) \equiv \int \frac{d^{3} \epsilon}{(2 \pi \hbar)^{3}} e^{\frac{i \mathbf{p} \cdot \epsilon}{\hbar}} \hat{\psi}_{\alpha^{\prime}}^{+}\left(\mathbf{r}+\frac{\epsilon}{2}\right) \hat{\psi}_{\alpha}\left(\mathbf{r}-\frac{\epsilon}{2}\right)$.

The Heisenberg equations are $\dot{\hat{W}}_{\alpha \alpha^{\prime}}(\mathbf{r}, \mathbf{p})=$ $\frac{i}{\hbar}\left[\hat{H}, \hat{W}_{\alpha \alpha^{\prime}}(\mathbf{r}, \mathbf{p})\right]$, where $\hat{H}$ is the Hamiltonian operator

$$
\begin{gathered}
\hat{H}=\sum_{j} \int d^{3} \mathbf{r} \hat{\psi}_{j}^{+}(\mathbf{r})\left[-\frac{\hbar^{2}}{2 m} \nabla^{2}+U_{j}+\hbar \omega_{j}\right] \hat{\psi}_{j}(\mathbf{r})+ \\
\sum_{j, k} \int d^{3} \mathbf{r}_{1} \int d^{3} \mathbf{r}_{2} \hat{\psi}_{j}^{+}\left(\mathbf{r}_{1}\right) \hat{\psi}_{k}^{+}\left(\mathbf{r}_{2}\right) u_{j k}\left(\mathbf{r}_{1}-\mathbf{r}_{2}\right) \hat{\psi}_{k}\left(\mathbf{r}_{2}\right) \hat{\psi}_{j}\left(\mathbf{r}_{1}\right)
\end{gathered}
$$

with $U_{j}=\frac{1}{2} m \omega_{\perp}^{2}\left(x^{2}+y^{2}\right)+\frac{1}{2} m \omega_{z j}^{2} z^{2}$ and $u_{j k}\left(\mathbf{r}_{1}-\mathbf{r}_{2}\right)=$ $\frac{1}{2} \hbar g_{j k} \delta\left(\mathbf{r}_{1}-\mathbf{r}_{2}\right)$. Here, $j, k$ refer to states $|1\rangle$ or $|2\rangle ; U_{j}$ is the total external potential (combined optical and magnetic) for atoms in state $j ; \hbar \omega_{j}$ is hyperfine energy of state $j ; g_{j k}=4 \pi \hbar a_{j k} / m$ with $m$ thes atomic mass. $a_{j k}$ is the scattering length for atoms between state $j$ and state $k$.

Spin density operators, $\hat{s}_{x}=\left[\hat{\psi}_{1}^{+}(\mathbf{r}) \hat{\psi}_{2}(\mathbf{r})+\right.$ H.c. $] / 2$, etc., can be written in terms of the momentum integral of the Wigner phase space operators,

$$
\int \hat{W}_{\alpha \alpha^{\prime}}(\mathbf{r}, \mathbf{p}) d^{3} \mathbf{p}=\hat{\psi}_{\alpha^{\prime}}^{+}(\mathbf{r}) \hat{\psi}_{\alpha}(\mathbf{r}) .
$$

The corresponding spin current operators are obtained using

$$
\int \frac{\mathbf{p}}{m} \hat{W}_{\alpha \alpha^{\prime}}(\mathbf{r}, \mathbf{p}) d^{3} \mathbf{p}=-\frac{i \hbar}{2 m} \hat{\psi}_{\alpha^{\prime}}^{+}(\mathbf{r}) \nabla \hat{\psi}_{\alpha}(\mathbf{r})+H . c .
$$

We obtain the spin-wave equations for both a Bose gas and a Fermi gas,

$$
\begin{gathered}
\frac{\partial S^{(i)}}{\partial t}+\nabla \cdot \mathbf{J}^{(i)}=(\boldsymbol{\Omega} \times \mathbf{S})^{(i)} \\
\frac{\partial \mathbf{J}^{(i)}}{\partial t}+\alpha \nabla S^{(i)}= \\
\left(\boldsymbol{\Omega}^{\prime} \times \overleftrightarrow{J}\right)^{(i)}-\gamma \mathbf{J}^{(i)} \\
-\frac{1}{2 m} S^{(i)} \nabla U-\frac{1}{4 m} n \nabla U^{\prime(i)},
\end{gathered}
$$

where

$$
\begin{gathered}
\boldsymbol{\Omega}=\left(\omega_{0}+\delta \omega_{1}+\delta \omega_{2}\right) \hat{z} \\
\delta \omega_{1}=\frac{1}{\hbar}\left(U_{1}-U_{2}\right), \\
\delta \omega_{2}=2 g_{11} n_{1}-2 g_{22} n_{2}-2(\epsilon+1) g_{12} S^{(z)}, \\
\boldsymbol{\Omega}^{\prime}=\mathbf{\Omega}+2 \epsilon g_{12} \mathbf{S} \\
U=U_{1}+U_{2}+2 \hbar g_{11} n_{1}+2 \hbar g_{22} n_{2}+\hbar g_{12} n, \\
\mathbf{U}^{\prime}=\hbar \mathbf{\Omega}^{\prime} .
\end{gathered}
$$

Here $i=x, y, z$ is the spin space index: $S^{(i)}$ is a spin density. For example, $S^{(z)}=\frac{1}{2}\left(n_{1}-n_{2}\right)$ with $n_{1}, n_{2}$ 


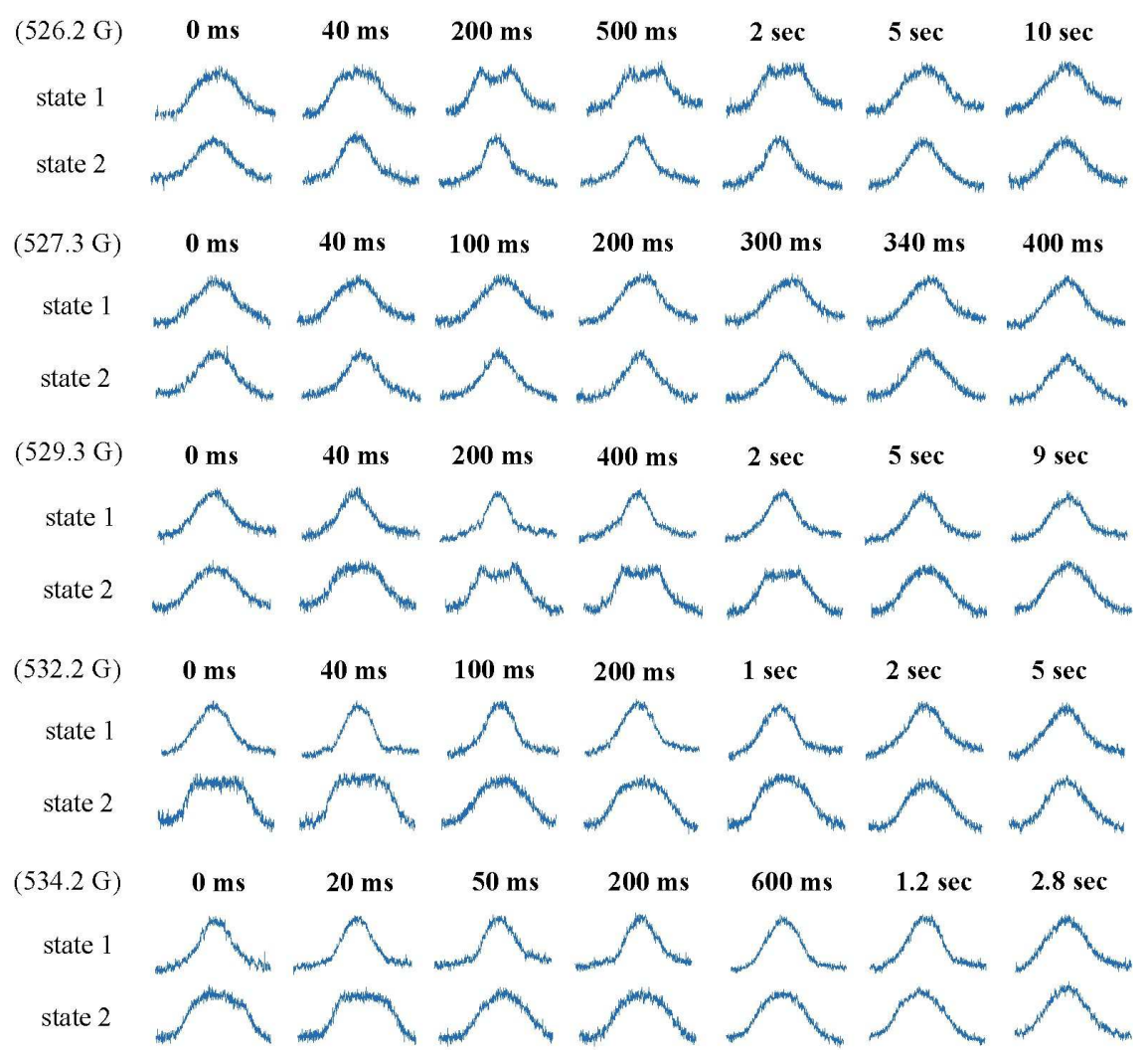

FIG. 2: Axial atomic density profiles (integrated over transverse directions) at various times after the RF pulse for different magnetic fields. The scattering length is estimated with $a(B)=\dot{a}\left(B-B_{0}\right)$, where $a ́=3.5 a_{0} / \mathrm{G}[\underline{8}$, 9$]$ and $B_{0}=527.5 \mathrm{G}$ (described below) Note that different graphs have different time scales.

the atom densities in states $|1\rangle$ and $|2\rangle . \mathbf{J}^{(i)}$ is the spin current corresponding to spin component $i[1]$. $\omega_{0} / 2 \pi$ is the hyperfine transition frequency. $\alpha=k_{B} T / m . n$ is the total atom number. $\gamma$ is the relaxation rate, which we take to be 0 in modelling the data. Finally, $\epsilon=+1(-1)$ for Bosons (Fermions) and $a_{11}=a_{22}=0$ for Fermions.

Numerical simulations based on these equations quantitatively reproduce prior calculations of spin segregation in Bose gases [4, 5, 6] that agree with the experiment. In contrast, these equations only qualitatively describe the spin segregation observed in our experiments with a Fermi gas. The equations correctly predict the inversion of the roles of the spins when the scattering length is reversed in sign. However, the predicted amplitude of the spin segregation derived from the calculations is two orders of magnitude smaller than what we observe; the predicted segregation time is one order of magnitude smaller than what we observe. This suggests that additional physics is important for explaining spin segregation in a Fermi gas.

Fig. 2 also shows the damping time (defined as the time for the cloud to relax back to its original density profile) increases as the scattering length is reduced in magnitude. It is interesting to note that for certain magnetic fields (for example, $526.2 \mathrm{G}$, where the scattering length is about $5 a_{0}, a_{0}$ is Bohr radius), spin segregation can be maintained for up to $5 \mathrm{~s}$. This result is strikingly longer than that observed in the previous experiment with a Bose gas, where the samples relaxed from maximum spin segregation back to their original density profiles in about $200 \mathrm{~ms}$. We attribute this result to the facts that ultracold fermionic ${ }^{6} \mathrm{Li}$ atoms interact only via $\mathrm{s}$-wave scattering between opposite spin states and that the ${ }^{6} \mathrm{Li}$ scattering length near the zero crossing is much smaller than for the Bose gas experiments, $\sim 5 a_{0}$ compared to $\sim 100 a_{0}$ for ${ }^{87} \mathrm{Rb}$.

We also note that the decoherence time in our experiment is measured to be about $70 \mathrm{~ms}$, which is comparable to the longest spin segregation time that we observe. The decoherence time is the measured time scale for the phase of the coherent superposition to decay, producing a simple binary mixture. Decoherence is caused by the spatial variation of the magnetic field across the sample. The short decoherence time may explain why we can not clearly observe spin segregation when the magnetic field is too close to the zero crossing. When the scattering length is very small, the time required for spin segregation to build up is longer than the decoherence time.

An additional experiment was performed to further study the spin segregation. After we create a sample 


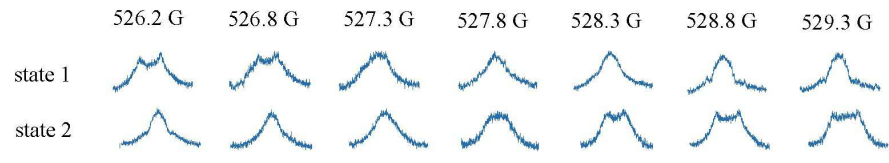

FIG. 3: Axial atomic density profiles at $200 \mathrm{~ms}$ after coherence is created by an RF pulse, for different magnetic fields. The zero crossing point in the scattering length is determined by studying the [transition-NOT a transition] in the spin segregation behavior as the magnetic field is varied.

that reaches the maximum spin segregation in $200 \mathrm{~ms}$ at $529 \mathrm{G}$, we immediately blow out atoms of one state using a resonance optical pulse. Then we take images of the remaining state at various times after the optical pulse. We observe that spin segregation maintains its atomic density profile for $\sim 2$ seconds. This shows that slow relaxation of one state does not depend on presence of the other state. As we know, a single-peak gaussian distribution is a stationary equilibrium state for a thermal cloud in a harmonic trap. In contrast, the double-peak and one-peak non-gaussian atomic density profiles observed in our experiments are far from equilibrium. It is remarkable that we can create a non-equilibrium stationary system that has such a long lifetime, $\sim 2 \mathrm{~s}$, compared to the axial trap period $6.9 \mathrm{~ms}$ and segregation time $\sim 200$ ms.

Previous experiments have shown that a zero crossing occurs in the $|1\rangle-|2\rangle$ scattering length of ${ }^{6} \mathrm{Li}$ near 528 $\mathrm{G}$ [12, 13]. We now describe a new determination of the zero crossing based on the behavior of the spin segregation as the magnetic field is varied. As described above, the roles of the spin states are interchanged as the scattering length $a$ changes sign from negative to positive. Fig. 2 shows data for $a<0$ at $526.2 \mathrm{G}$ and for $a>0$ at 529.3 G. The spin segregation time (defined as the time for the cloud to reach maximum spin segregation) decreases (increases) as the scattering length increases (decreases). Spin segregation becomes difficult to observe when the magnetic field is too close to the zero crossing, for example, $527.3 \mathrm{G}$ in the figure, as the segregation time then far exceeds the decoherence time, as noted above. Similarly, very far from the zero crossing, $\sim 15 \mathrm{G}$ above or below collisional relaxation dominates and spin segregation is not observable (not shown in the figure).

Taking advantage of the fact that spin segregation slows for magnetic fields very close to the zero crossing, we determine the zero crossing precisely. We take absorption images of both states at $200 \mathrm{~ms}$ after the RF pulse for different magnetic fields. Fig. 3 shows a clear change in the spin segregation behavior from $526.2 \mathrm{G}$ to $529.3 \mathrm{G}$. The magnetic fields are calibrated by RF spectroscopy using the Hamiltonian for the ${ }^{6} \mathrm{Li}$ ground state hyperfine interactions in a magnetic field,

$$
H_{i n t}=\frac{a_{h f}}{\hbar^{2}} \mathbf{S} \cdot \mathbf{I}-\frac{\mu_{B}}{\hbar}\left(g_{J} \mathbf{S}+g_{I} \mathbf{I}\right) \cdot \mathbf{B},
$$

where $\mu_{B} / h=1.399624604 \mathrm{MHz}$ is the Bohr magneton, $a_{h f} / h=152.1368407 \mathrm{MHz}$ [14] is the ground state magnetic hyperfine constant for ${ }^{6} \mathrm{Li}, g_{J}=-2.0023010$ [14] is the total electronic g-factor for the ${ }^{6} \mathrm{Li}$ ground state, $g_{I}=0.0004476540$ [14] is the nuclear g-factor, $h$ is Planck's constant, and B is the bias magnetic field.

The transition in the behavior of the segregation occurs at $527.5 \mathrm{G}$, which is determined as follows. We fit the axial atomic density profiles of both states with a gaussian distribution for the data taken at $527.3 \mathrm{G}$ and 527.8 Gs. For each data set, we derive gaussian widths of both clouds and their ratio. The ratio of the width of state $|1\rangle$ to that of state $|2\rangle$ is greater than 1 for $527.3 \mathrm{G}$; less than 1 for $527.8 \mathrm{G}$. By interpolation, we find the point where the ratio of widths is equal to 1 , which determines the value of magnetic field $527.5 \pm 0.2 \mathrm{G}$ (the error bar is the quadratic combination of the shot-to-shot fluctuation of the magnetic field setting and the magnetic field calibration error arising from the RF spectrum fit). Since no spin segregation occurs with the scattering length of zero, we interpret the value of magnetic field where the ratio of widths is 1 to be the zero crossing.

In conclusion, we have observed spin segregation in a trapped ultracold atomic Fermi gas of ${ }^{6} \mathrm{Li}$ with a scattering length close to zero. The observed behavior of the spin segregation appears to require a modification of the current theory based on spin waves, or possibly a new mechanism. In addition, using spin segregation, we have precisely determined the zero crossing in the scattering length of ${ }^{6} \mathrm{Li}$.

This research is supported by ARO, NSF, and DOE.

* jet@phy.duke.edu

[1] E. Tiesinga, A. J. Moerdijk, B. J. Verhaar, and H. Stoof, Phys. Rev. A 46, R1167 (1992); E. Tiesinga, B. J. Verhaar, and H. Stoof, Phys. Rev. A 47, 4114 (1993).

[2] K. M. O'Hara et al., Science 298, 2179 (2002).

[3] S. Giorgini, L. P. Pitaevskii, and S. Stringari, arXiv: 0706.3360 (2007), to appear in Rev. Mod. Phys.

[4] M. Ö Oktel and L. S. Levitov, Phys. Rev. Lett. 88, 230403 (2002).

[5] J. E. Williams, T. Nikuni, and Charles W. Clark, Phys. Rev. Lett. 88, 230404 (2002).

[6] J. N. Fuchs, D. M. Gangardt, and F. Laloë, Phys. Rev. Lett. 88, 230405 (2002).

[7] H. J. Lewandowski et al., Phys. Rev. Lett. 88, 070403 (2002).

[8] M. Bartenstein et al., Phys. Rev. Lett. 94, 103201 (2005).

[9] P. S. Julienne, private communication.

[10] J. E. Thomas and L. J. Wang, Phys. Rep. 262, 311 (1995).

[11] $\overleftrightarrow{J}$ is a tensor and $\mathbf{J}^{\mathbf{i}}$ is a vector in coordinate space.

[12] K. M. O'Hara et al., Phys. Rev. A 66, 041401(R) (2002).

[13] S. Jochim et al., Phys. Rev. Lett. 89, 273202 (2002).

[14] E. Arimondo, M. Inguscio, and P. Violino, Rev. Mod. Phys. 49, 31 (1977). 